\title{
Le SAGE nappes profondes de la Gironde
}

\author{
The deep groundwater SAGE in Gironde \\ par H. Servat et B. Gaillard \\ Direction Régionale de l'Environnement d'Aquitaine
}

Growing needs for drinking water, but also for industry and agriculture, lead to a critical situation of deep ground water resources around Bordeaux. Though several decades of close attention, water quality is threatened by a risk of saline intrusion from the Gironde estuary into Eocene ground water. All involved partners, including local governments and directly concerned users, take part in the elaboration of a water management plan, that would strike a balance between resources and needs.

\section{I $\square$ LE SAGE : L'ABOUTISSEMENT D'UN DIAGNOSTIC ET DE MUL- TIPLES DÉMARCHES}

$\mathrm{Au}$ chapitre des règles de protection particulières des eaux souterraines (mesure C21), le Schéma Directeur d'Aménagement et de Gestion des Eaux Adour-Garonne recommande dans les deux ans à compter de l'approbation du SDAGE que les services de l'Etat et les collectivités concernées avec l'appui de l'Agence de l'Eau recherchent dans la région centrée sur Bordeaux des ressources alternatives à celles de l'Eocène afin d'en préserver la qualité (risques d'intrusion saline et d'extension du secteur minéralisé), en présentent l'inventaire à la commission nappes profondes, et mettent en œuvre ces solutions en tant que de besoin. Il sera tenu compte pour cette orientation des conclusions du schéma directeur de gestion de la ressource en eau du département de la Gironde.

A partir de cette recommandation de protection et d'exploitation des eaux souterraines, a été engagée la démarche du Schéma d'Aménagement et de Gestion des Eaux (SAGE) "Nappes profondes" de la Gironde. Le texte qui suit se propose de présenter la problématique spécifique de l'Eocène ainsi que les différentes phases et acteurs qui ont contribué au suivi des nappes profondes et aux diagnostics initiaux et contribuent maintenant à la mise en œuvre de ce SAGE.

\section{II $\square$ LE CADRE HYDROGÉOLOGIQUE}

Le bassin aquitain est constitué d'une superposition de couches perméables et imperméables des contreforts du Massif Central jusqu'au talus continental de l'océan Atlantique. Cette configuration géologique favorise l'infiltration des eaux météorites sur les affleurements des couches à l'extérieur du département et leur transfert souterrain dans le sous-sol girondin vers les exutoires naturels de l'estuaire de la Garonne et de l'océan.
Les systèmes aquifères profonds en Gironde appartiennent à des formations géologiques allant du Jurassique (250 millions d'années) au Pliocène (1 million d'années). On distingue :

- le Pliocène (système $n^{\circ} 234$ ) à l'ouest du département, situé entre 30 et 80 mètres de profondeur, dont l'usage est limité le plus souvent à l'irrigation,

- le Miocène (système $n^{\circ} 235$ ) à l'ouest du département, situé entre 20 et 150 mètres de profondeur, utilisé pour l'eau potable et l'irrigation,

- l'Oligocène (système $\mathrm{n}^{\circ}$ 230) à l'ouest de la Garonne entre 50 et 500 mètres de profondeur et les plateaux calcaires de l'Entre-Deux-Mers, alimentant des sources importantes captées pour l'eau potable et exploité également par forage pour l'AEP,

- l'Eocène (système $n^{\circ} 214$ ) qui couvre la quasi-totalité du territoire et se situe entre 100 et plus de 500 mètres de profondeur ; il est utilisé principalement pour l'alimentation en eau potable, mais aussi par les industries dans la presqu'île d'Ambès et quelques irriguants près des affleurements,

- le Crétacé (systèmes n 231 et 215 ) qui s'étend sur la totalité du département et se situe entre 300 et 1100 mètres de profondeur : il est exploité pour l'eau potable, l'industrie (forages de secours de la centrale nucléaire du Blayais) et la géothermie,

- le Jurassique (système $\mathrm{n}^{\circ} 217$ ) qui se situe entre 800 et 1500 mètres de profondeur sous tout le territoire avec des débits exploitables importants mais des eaux chaudes et souvent minéralisées.

La nappe de l'Eocène est alimentée par les précipitations sur les zones d'affleurement en marges du bassin qui contribuent également à sa mise en pression, par le réseau hydrographique dont la Dordogne à l'est de Bergerac et également par drainance des eaux des nappes sus-jacentes (Oligocène du Médoc) et sous-jacentes. Les exutoires naturels se situent principalement au niveau de l'estuaire et de l'océan. 


\section{L'ÉVOLUTION DES PRÉLÈVEMENTS DANS LES NAPPES PROFONDES}

\subsection{La répartition par nappes et par usages}

De 1970 à 1998, les volumes annuels prélevés dans les aquiferes profonds sont passés de 88 à 147 millions de $\mathrm{m}^{3}$. En 1998, on dénombrait 832 captages en exploitation avec la répartition en volume suivante (figure 1) :

\author{
- $13 \%$ pour le Miocène, \\ - $42 \%$ pour l'Oligocène, \\ - $40 \%$ pour l'Eocène, \\ - $5 \%$ pour le Crétacé, \\ et celle par usages ci-après (figure 2 ) : \\ $-74 \%$ pour l'AEP, \\ - $17 \%$ pour l'irrigation, \\ - $5 \%$ pour l'industrie, \\ - $3 \%$ pour la géothermie, \\ $-1 \%$ pour divers usages.
}

\subsection{La répartition par usages des prélèvements dans l'Eocène}

De 1970 à 1998, les prélèvements dans l'Eocène supérieur ont progressé de 1,6 à 4 millions de $\mathrm{m}^{3}$ dont 2,9 millions pour l'AEP, 0,4 million pour l'industrie (entre 1974 et 1984, ce chiffre a fluctué entre 0,7 et 1,5 million de $\mathrm{m}^{3}$ ) et 0,6 million pour l'agriculture.

Sur la même période, ceux de l'Eocène moyen à inférieur ont augmenté de 1970 jusqu'en 1989 de moins de 40 millions à 61,6 millions, puis ont subi des fluctuations pour atteindre en 1998 un volume de 54,4 millions de $\mathrm{m}^{3}$ se répartissant en (figures 3 et 4 ) :

- $86 \%$ pour l'AEP,

- $7 \%$ pour l'industrie,

- $3 \%$ pour l'agriculture,

- $3 \%$ pour la géothermie.

La production d'eau potable représente donc l'usage principal des nappes profondes en Gironde et plus particulièrement de l'aquifère de l'Eocène.

\subsection{L'impact des prélèvements sur l'Eocène}

Alors que les forages captant l'Eocène étaient à l'origine artésiens, l'accroissement des prélèvements à partir des années 1950 a provoqué rapidement un abaissement des pressions de la nappe dans les zones les plus sollicitées. Ainsi, de 1950 à 1960, la cote piézométrique est passée de +10 à +5 mètres NGF sous Bordeaux.

En 1959, ces observations alarmantes ont abouti pour l'ensemble de la Gironde, à l'application du décret-loi de 1935 applicable aux nappes artésiennes du bassin parisien. Cette réglementation qui impose une procédure d'autorisation pour tout prélèvement dans un forage de plus de 60 mètres, a permis de disposer d'un suivi des nappes profondes et en particulier de l'Eocène.

En 1970, du fait de l'augmentation des prélèvements, notamment industriels, sur le bec d'Ambès, le surcreusement s'accentue jusqu'à des niveaux de -5 et -10 mètres NGF et la dépression s'élargit. A la fin des années 1980, des cotes de -20 mètres NGF sont atteintes. Le cycle de sécheresse 1989-1992 provoque de nouveau une accentuation de la dépression sous Bordeaux. Enfin, depuis 1996, la baisse des prélèvements dans l'Eocène sous Bordeaux au profit de nou-

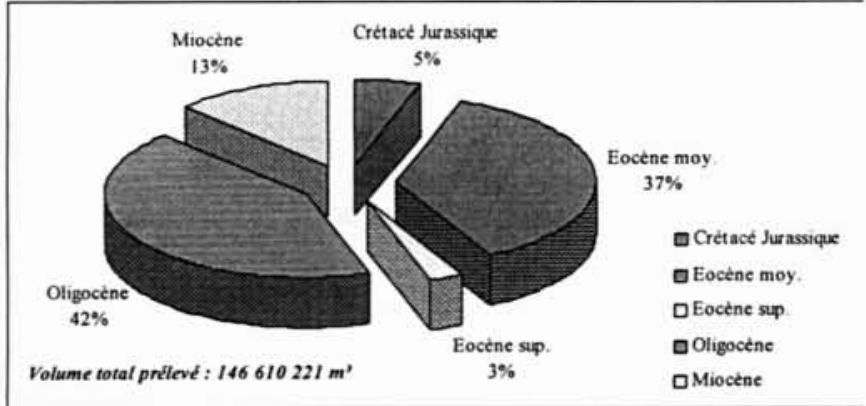

1. Répartition en $\mathbf{1 9 9 8}$ des volumes prélevés en fonction du système aquifère capté (rapport BRGM / SGR Aquitaine - $\mathrm{R}$ 40844).

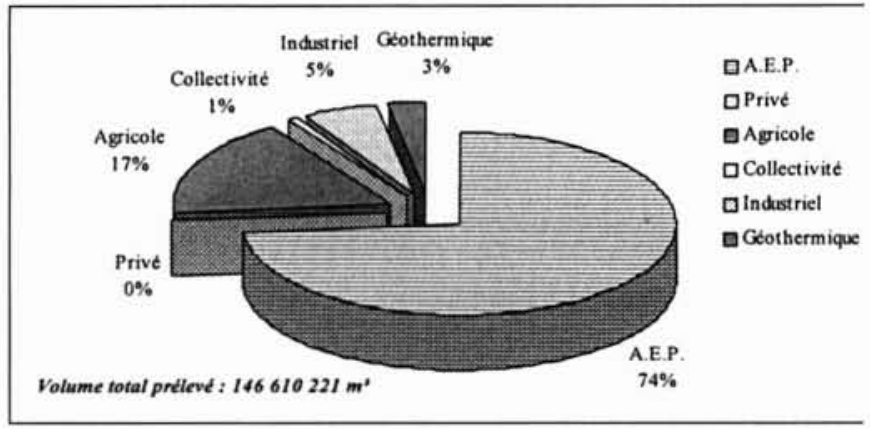

2. Répartition en 1998 des volumes prélevés en fonction de l'usage de l'eau ((rapport BRGM / SGR Aquitaine R 40844).

veaux prélèvements dans la même nappe à l'extérieur de la zone ou bien dans l'Oligocène induit quelques remontées des niveaux mais aussi et surtout un déplacement du creux piézométrique vers l'est et le sud-est.

Ainsi depuis plus de quarante ans, on observe une accentuation progressive du creux piézométrique sous Bordeaux de l'ordre de 50 mètres. Les courbes de variations comportent cependant des ralentissements dus aux effets conjugués des activités humaines et des conditions climatiques, ce qui souligne la capacité de l'Eocène à restituer des variations positives si l'on stabilise les prélèvements. D'autre part, les nouveaux prélèvements sur d'autres nappes ou d'autres secteurs géographiques provoquent un élargissement et un déplacement du creux piézométrique.

Deux autres menaces pèsent sur cette nappe. Il s'agit en premier lieu de la présence d'un chenal minéralisé allant de Grignols au Médoc en passant par 1'Entre-Deux-Mers avec des teneurs supérieures à $250 \mathrm{mg} / \mathrm{l}$ et un taux de fluor supérieur à $1,5 \mathrm{mg} / \mathrm{l}$ pour certains forages. En second lieu, il existe un risque d'intrusion d'eaux saumâtres provenant de l'estuaire de la Gironde dans le système aquifère de l'Eocène moyen à inférieur.

\section{IV — LES OUTILS DE GESTION}

\subsection{Sur le plan technique}

Comme indiqué dans le paragraphe précédent, l'application du décret-loi de 1935 a permis au département de la Gironde d'être équipé d'un réseau dense de suivi des nappes profondes et plus particulièrement de l'Eocène. On dispose de ce fait de données anciennes de référence et d'une chronique importante de piézométrie.

Depuis les années 1960, de nombreuses études ont été 


\begin{abstract}
3. Evolution entre 1970 et 1998 des volumes prélevés (Eocène moyen à inférieur) en fonction de l'usage de l'eau (rapport BRGM / SGR Aquitaine - R 40844).
\end{abstract}

\section{Répartition en 1998 des volumes prélevés en fonction de l'usage de l'eau (rapport BRGM / SGR Aquitaine - R 40844)}

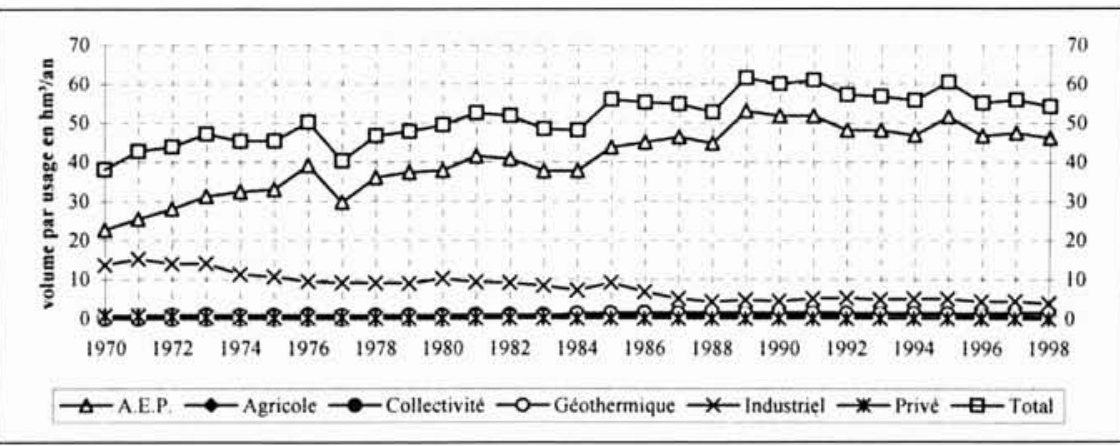

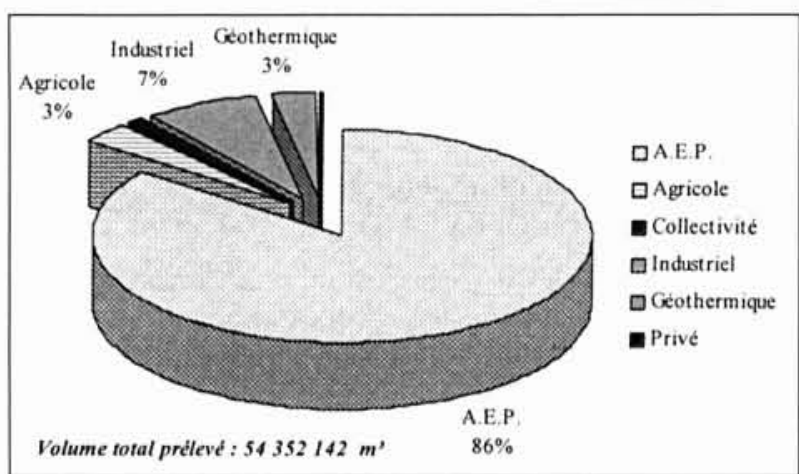

menées par le BRGM sur ces aquifères. Dans le cadre de financement d'accords-cadres et de conventions, des suivis annuels sont réalisés sur le plan quantitatif et qualitatif.

Une modélisation des quatre unités géologiques (Miocène, Oligocène, Eocène et Crétacé) a été menée par le BRGM, financée par l'Etat et l'Agence de l'Eau. Le modèle mathématique nord-aquitain couvre la totalité du département de la Gironde, le sud des Charentes, le sud-ouest de la Dordogne, le nord-ouest du Lot-et-Garonne et le nord des Landes. Suite à une expertise, le BRGM a entrepris en 1998 la restructuration du modèle avec cinq couches aquifères représentées : le Miocène-Plioquaternaire, l'Oligocène, l'Eocène, le Campanien-Maastrichtien (Crétacé terminal) et le Turonien-Santonien (Crétacé supérieur) qui constitue le "fond" du modèle.

Les résultats du modèle indiquent que :

- l'Oligocène est globalement en équilibre,

- l'Eocène est déficitaire d'environ $30000 \mathrm{~m}^{3} /$ jour,

- le Campanien est légèrement déficitaire de 3 à $4000 \mathrm{~m} 3 /$ jour.

A partir de ce modèle actualisé, six scénarios ont été simulés. Les scénarios 1 et 2 sont les suivants :

- scénario 1 "de référence" avec le maintien de prélèvements constants entre 1996 et 2010, quels que soient la couche du modèle et le secteur (concerne les trois départements),

- scénario 2 "dur" avec une augmentation de $11 \%$ des prélèvements AEP jusqu'en 2010, soit un accroissement de $0,8 \%$ par an, avec un maintien des prélèvements industriels et agricoles.

Les quatre autres scénarios présentent des hypothèses intermédiaires avec la prise en compte de diminutions sectorielles des prélèvements et la mise en œuvre de solutions de substitution.

Les résultats de ces deux simulations indiquent que le scénario 1 conduit à une aggravation du creux piézométrique mais aussi de la situation sur l'estuaire avec une baisse locale de 0,5 à 1 mètre. Le scénario 2 aboutit à des baisses de 0,7 à plus de 3 mètres sur l'estuaire, ce qui impliquerait un déséquilibre entre l'eau salée et l'Eocène.

\subsection{Les structures et les partenaires}

Le 16 mai 1991 a été mis en place un comité de gestion des eaux souterraines en Gironde. Cette structure initiée par l'Etat regroupe l'ensemble des acteurs de la gestion de la ressource, c'est-à-dire les services de l'Etat, les établissements publics, les collectivités territoriales, les sociétés de distribution et les universitaires. Elle constitue une instance de réflexions et d'orientations sur l'ensemble des problématiques liées aux eaux souterraines. Elle a permis notamment la réalisation d'une plaquette d'information diffusée en 5000 exemplaires, suscité la prise de conscience de la nécessité d'une concertation interdépartementale avec les départements de Dordogne et de Lot-et-Garonne et a activement participé à la création du schéma directeur d'aménagement et de gestion de la ressource en eau de la Gironde.

C'est en 1995 que le Conseil Général de la Gironde s'est porté maitre d'ouvrage de cette étude très générale sur les ressources et besoins en eaux jusqu'à l'horizon 2015. Les objectifs de ce schéma sont :

- de mener une gestion cohérente de la ressource en assurant sa pérennité,

- d'aboutir à l'adéquation entre la qualité de la ressource et les différents usages de l'eau,

- de donner une priorité à l'usage domestique de l'eau,

- de réserver les eaux souterraines de qualité à l'alimentation en eau potable,

- de garantir la sécurité de l'approvisionnement.

Cette structure a permis de réaliser de nombreuses études basées en particulier sur les modélisations mathématiques des ressources citées auparavant, préconisant de nouvelles mesures de gestion, dont la mise en œuvre de ressources de substitution.

A cet effet a été instauré en 1998, entre la Communauté Urbaine de Bordeaux (CUB) et le Conseil Général de la Gironde, un syndicat mixte d'étude pour la gestion de la ressource en eau du département de la Gironde. Cette création a été jugée cohérente avec la perspective d'un SAGE et la constitution d'une commission locale de l'eau. Une des premières démarches de ce syndicat a été d'engager le recalage du modèle mathématique du BRGM afin de disposer de résultats de simulations actualisés et validés.

\section{V — LE SAGE "NAPPES PROFONDES" DE LA GIRONDE}

\subsection{Les fondements du SAGE}

Les fondements reposent d'une part sur les constats décrits ci-avant et d'autre part sur les partenaires engagés dans la gestion des eaux souterraines. Parmi ceux-ci, on peut citer : 
- le Conseil Général de la Gironde et la Communauté Urbaine de Bordeaux (CUB) qui de par le schéma directeur de la gestion des eaux du département pour le premier cité et le schéma directeur de la CUB ont engagé très tôt des actions qui ont abouti à la création d'un syndicat mixte d'études et à la sollicitation commune du préfet de la Gironde pour la mise en place d'un SAGE,

- la Région Aquitaine qui a affiché une politique de l'eau dans le cadre du programme régional de gestion des eaux souterraines,

- l'administration de l'Etat avec la Mission Inter-Services de l'Eau de la Gironde, la Direction Régionale de l'Industrie de la Recherche et de l'Environnement, et la Direction Régionale de l'Environnement d'Aquitaine,

- l'Agence de l'Eau Adour-Garonne avec notamment la maîtrise d'ouvrage de nombreuses opérations,

- le BRGM qui a réalisé les suivis et les études sur les nappes profondes, dont la modélisation, et qui a attiré l'attention sur la surexploitation de la nappe de l'Eocène dans les publications et la présentation des résultats,

- et enfin, les représentants industriels, agricoles, les associations de protection de la nature,...

Au stade des connaissances acquises en 1997 et des solutions envisagées, il est apparu indispensable de disposer d'un outil juridique adapté permettant d'asseoir les préconisations techniques de gestion à mettre en œuvre. Le schéma d'aménagement et de gestion des eaux s'est révélé être l'outil le plus adapté. C'est ainsi que les procédures du SAGE nappes profondes de la Gironde ont été engagées à la fin de l'année 1997.

\subsection{Les différentes étapes de la phase préliminaire du SAGE}

Trois étapes importantes ont été franchies avant la consultation des collectivités. Il s'agit de la lettre conjointe du 3 septembre 1997 de la CUB et du Conseil Général de la Gironde demandant au préfet la mise en place du SAGE, de la présentation le ler décembre 1997 au comité de bassin Adour-Garonne du rapport Mahenc sur la surexploitation de l'Eocène et la proposition de résolution d'un moratoire sur l'Eocène, et enfin la présentation du sujet à la commission géographique nappes profondes du 20 janvier 1998.

Le 20 mars 1998, le préfet de la Gironde a engagé la consultation des collectivités locales (Conseils Régional et Général, communes concernées) pour recueillir leur avis sur le périmètre proposé qui était celui du département de la Gironde où se situe la grande majorité des prélèvements, l'abaissement piézométrique critique, le risque majeur d'intrusion saline pour l'alimentation en eau potable de l'agglomération bordelaise et, enfin, les différentes ressources de substitution et les projets d'équipement.

Les collectivités concernées ont émis un avis favorable à cette délimitation avec cependant une demande en particulier du Conseil Régional et des départements de Dordogne et du Lot-et-Garonne pour que soit envisagée rapidement une extension de ce périmètre aux contours des aquiferes.

Le 10 juin 1998, le Préfet de la Gironde a saisi le Préfet coordonnateur de bassin sur les limites du SAGE et la composition de la commission locale de l'eau.

Le 6 juillet, le comité de bassin Adour-Garonne a donné un avis favorable à la délimitation du périmètre sous réserve de la création d'un groupe de liaison (conformément à la mesure F4 du SDAGE) chargé notamment de suivre les travaux de la
Commission Locale de l'Eau (CLE) et d'un élargissement du SAGE aux zones d'extension des systèmes aquifères dès que les études complémentaires seraient réalisées.

Le périmètre du SAGE a été fixé à l'ensemble du territoire du département de la Gironde par arrêté préfectoral du 19 août 1998. La décision de ne pas intégrer d'emblée les départements voisins a été dictée par la volonté d'agir vite.

Enfin, la composition de la commission locale de l'eau a été fixée par arrêté du 22 mars 1999. La CLE est composée d'un collège des représentants des collectivités territoriales et des établissements publics locaux, d'un collège des représentants des usagers, des organisations professionnelles et des associations concernées, d'un collège des représentants de l'Etat et de ses établissements publics et pour finir d'experts et de personnes associées.

\subsection{Les premiers travaux de la phase d'élaboration du SAGE}

Afin de mener les six séquences de la conception du projet de SAGE, trois groupes de travail ont été créés : groupes de travail "quantité", "qualité" et "économies d'eau". La première étape a consisté à dresser l'état des lieux technique et réglementaire des connaissances. La démarche menée en collaboration avec le BRGM en tant qu'expert a fait l'objet de nombreuses réunions de travail et de présentations successives à la CLE.

La recherche d'un prestataire pour la mission d'animation et de réalisation du SAGE a abouti en début d'année 2000. Le bureau d'études retenu a eu comme première mission de coordonner les résultats des trois groupes de travail et d'élaborer un document de synthèse de l'état des lieux qui a été présenté pour validation à la CLE réunie en avril.

L'étape suivante consistera à établir le diagnostic global avant d'aborder les tendances et scénarios, le choix de la stratégie, les produits du SAGE (orientations de gestion et d'aménagement) et pour terminer cette première partie, la validation finale par la CLE. Lors de cette phase devront être en particulier définis les objectifs de protection quantitative et qualitative des eaux souterraines. Pour chaque système aquifère, cela se traduira par le choix de points d'observation auxquels seront associées des valeurs de piézométrie objectif d'étiage (POE) assurant la coexistence normale des usages et de piézométrie de crise (PCR) au-dessous de laquelle est mise en péril la pérennité de la ressource avec toutes les conséquences qui en découlent.

Le Conseil Général de la Gironde assure la maîtrise d'ouvrage de ces études préalables.

\section{VI $\square$ CONCLUSIONS}

Le SAGE nappes profondes de la Gironde va permettre à terme de disposer d'un outil de gestion indispensable pour garantir la pérennité de la ressource en eaux souterraines. Sa mise en œuvre bénéficie de connaissances scientifiques importantes et peut s'appuyer sur l'ensemble des partenaires impliqués dans les démarches initiales.

Cet aspect positif implique cependant un équilibre fragile à rechercher entre la prise en considération des solutions de substitution déjà définies dans le schéma directeur d'aménagement et la nécessaire démarche méthodologique du SAGE.

L'achèvement des travaux auxquels seront associés les départements voisins au sein du groupe de liaison est fixé selon le calendrier à la fin de l'année 2000. 